ORIGINAL ARTICLE

\title{
Lead the way or leave the way: Early experience of management of emergency pelvi-acetabular surgery during the COVID-19 pandemic at a tertiary care hospital in Pakistan.
}

Umair Nadeem ${ }^{1}$, Atif Ali², Nayab Shakoor ${ }^{3}$, Jawad Hafeez ${ }^{4}$, Majid Zaheer $^{5}$, Zubair Khalid ${ }^{6}$, Ameer Aziz $^{7}$

Article Citation: Nadeem U, Ali A, Shakoor N, Hafeez J, Zaheer M, Khalid Z, Aziz A. Lead the way or leave the way: Early experience of management of emergency pelvi-acetabular surgery during the COVID-19 pandemic at a tertiary care hospital in Pakistan. Professional Med J 2022; 29(3):291-296. https://doi.org/10.29309/TPMJ/2022.29.03.6569

ABSTRACT... Objective: To report the early experience, the relationship of surgical site infection after pelvi-acetabular fracture fixation with certain possible risk factors, and organizational protocol for emergency pelvic surgery currently being used during the COVID-19 pandemic by an integrated team of orthopedic surgeons. Study Design: Prospective study. Setting: Department of Orthopedic and Spine Surgery, "Ghurki Trust Teaching Hospital Lahore, Pakistan. Period: March 2020, to August 2020. Material \& Methods: A total of 25 patients diagnosed with acetabular fractures were included. Fracture patterns were classified according to judet and letournel and young burgess classification systems. Factors studied included patient's age, gender, body mass index, fracture pattern, type of surgery, comorbidities, smoking status, associated injuries, surgical site infections and mortality. Results: In a total of 25 patients, $21(84.0 \%)$ were male and $4(16.0 \%)$ female patients with overall mean age as $38.04 \pm 14.58$ years. Overall, mean BMI was $23.20 \pm 2.60 \mathrm{~kg} / \mathrm{m}^{2}$. There were $12(48.0 \%)$ patiens who had hypertension while $4(16.0 \%)$ smokers. There were $21(84.0 \%)$ patients who did not have any associated injuries while $3(12.0 \%)$ had the associated extremities. The mean hospital stay of the patients was $5.08 \pm 0.76$ days. The results revealed no significant association of Gender with these parameters $(p>0.001)$. Conclusion: There is no added risk of infection and mortality for the provision of emergency trauma services to pelvi-acetabular fractured patients even during panic and pandemic situations.

Key words: $\quad$ COVID-19, Pelvi-Acetabular, Surgical Site Infection.

\section{INTRODUCTION}

On 18 March 2020, novel coronavirus (COVID-19) patients had been registered in all over Pakistan. ${ }^{1}$ Pakistan currently has the fourth-largest number of confirmed cases in Asia, the second-largest number of confirmed cases in South Asia, coming after India, and the 15th highest number of confirmed cases in the world. ${ }^{2}$ One study from wuhan city showed that the peak date of onset of orthopaedic surgeons' infection was 8 days earlier than that of the public, indicating that orthopedic surgeons were more likely exposed to COVID-19 in the hospitals, rather than in the community. ${ }^{3}$ The high rate $(20.8 \%)$ of transmission to family members raised doctors' need to be cautious of household transmission. ${ }^{3}$
Orthopedic surgeons had to be more vigilant and take more precautions to avoid infection with COVID-19. The global nature of the threat, the enemy's invisibility, and the unpredictable target has created the perfect storm. Globally, a chaotic response was observed in hospitals. Panic situation was created. One week later, "American Academy of Orthopaedic Surgeons (AAOS)" issued triage that recommended only acute injuries and malignancies to be treated and delaying elective surgeries advocated by the "Centers for Medicare and Medicaid Services (CMS)", "The American College of Surgeons (ACS)", and the U.S. Surgeon General. ${ }^{4}$

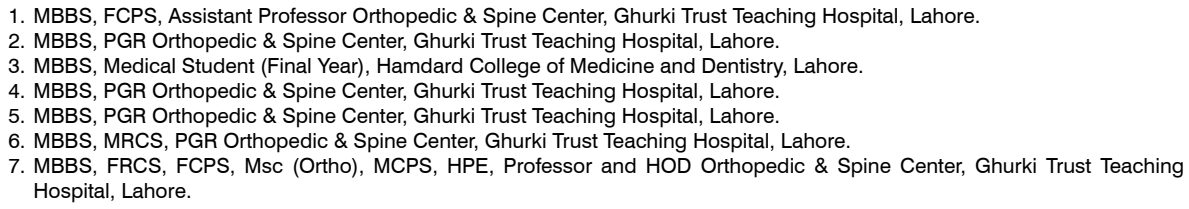

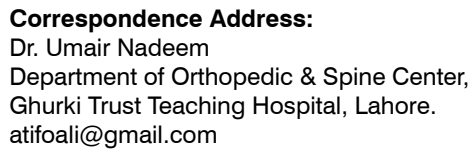

Article received on: Accepted for publication: 
Pelvic fractures are considered to be the $3^{\text {rd }}$ most frequent cause of death from road-traffic accidencts (RTAs) while mortality in between $5-15 \% .^{5}$ Recent advancements in surgical treatment of pelvic ring fractures have enchanced outcomes and survival of the individuals affected. Acute open reduction and internal fixation (ORIF) of unstable pelvic ring fractures showed a trend toward benefit compared with a staged approach. ${ }^{5}$ Delaying the fixation increases the difficulty of obtaining an anatomic reduction. . $^{6-11}$

Due to the emergent nature of pelvi-acetabular trauma, we decided to continue our services despite health threats. The closure of health care facilities in other hospitals in the country put an additional load on our hospital. This research was aimed to report the early experience, surgical site infection (SSI) relationship after pelvi-acetabular fracture fixation with certain possible risk factors, and organizational protocol for emergency pelvic surgery currently being used during the COVID-19 pandemic by an integrated team of orthopedic surgeons.

\section{MATERIAL \& METHODS}

A prosspective study was conducted at the Department of Orthopedic and Spine Surgery, "Ghurki trust teaching hospital (GTTH) Lahore, Pakistan". The hospital ethical review committee approved the study (2021/04/R-21) (REEDS). Patients included in the study were operated for Acetabulum and pelvic ring fractures between March 19, 2020, and August 13, 2020, during the COVID-19 pandemic. Patients with a head injury, hemodynamically unstable patients, patients managed conservatively/primary hip replacement, and open fractures were excluded from the study. Fracture patterns were classified according to judet and letournel and young burgess classification systems. ${ }^{12,13}$

Factors studied included patient's age, gender, BMI, fracture pattern, type of surgery, patient's comorbidities (diabetes mellitus, hypertension, ischemic heart disease, chronic obstructive pulmonary disease, and cirrhosis), smoking status, associated injuries, SSI and mortality. All the patients with Pelvi-acetabular trauma were screened for COVID-19 by measuring body temperature, chest Xrays, travel and contact records, and clinical signs and symptoms in the hospital emergency department. This pelviacetabular team performed each surgery. Administration of prophylactic antibiotics were done 30 minutes prior to the start of the procedures. Surgeries were performed in modular operation theaters of GTTH after disinfecting the skin with chlorhexidine solution, and disposable drapes were used. Patients were examined for surgical site infections daily during the hospital stay by the same team, and follow-up was done in the outpatient department at 2 and 6 weeks.

The definition of SSI was based on Southampton Wound Assessment Scale. ${ }^{14}$ Grade I, II, were not considered infections and grade III,IV,V and VI were taken as infections when it occurred beneath the muscle's incision area and the muscle's tissues. Mortality was recorded as patient's death from $1^{\text {st }}$ day of surgery to 4 months following surgery, either as inpatient or outpatient. The data was analyzed using IBM SPSS version 26.0. Qualitiave data like gender, comorbidities, associated injuries, site and grading of fracture were highlighted as frequency and percentages. Quantitative data like age, BMl and duration of hospital stay were highlighted as mean and standard deviation. Post stratification chi square test was applied to see if there was any gender bases difference amaong patiens considering $p$ value $<0.05$ as significant.

\section{RESULTS}

In a total of 25 patients, $21(84.0 \%)$ were male and $4(16.0 \%)$ female. Overall, mean age was $38.04 \pm 14.58$ years (ranged from 14-75 years) while mean BMI was $23.20 \pm 2.60 \mathrm{~kg} / \mathrm{m}^{2}$ (ranged from $18-28 \mathrm{~kg} / \mathrm{m}^{2}$ ). Out of 25 patients, $12(48.0 \%)$ were associated with hypertension, while $4(16.0 \%)$ were smokers. Three patients (12.0\%) had the associated extremities followed by genitourinary $(n=1,4 \%)$ while the majority $21(84.0 \%)$ did not have any associated injuries (Table-I). The mean stay of patients in the hospital was $5.08 \pm 0.76$ days. 


\begin{tabular}{|l|c|c|}
\hline \multicolumn{2}{|c|}{ Characteristics } & Frequency (\%) \\
\hline \multirow{3}{*}{ Gender } & Male & $21(84.0)$ \\
\hline \multirow{4}{*}{ Comorbidity } & Female & $4(16.0)$ \\
\cline { 2 - 3 } & Smokers & $4(16.0)$ \\
\cline { 2 - 3 } & $\begin{array}{c}\text { Chronic obstructive } \\
\text { pulmonary disease } \\
\text { (COPD) }\end{array}$ & $1(4.0)$ \\
\cline { 2 - 3 } & $\begin{array}{c}\text { Hypertension } \\
\text { Nil }\end{array}$ & $12(48.0)$ \\
\hline \multirow{3}{*}{$\begin{array}{c}\text { Associated } \\
\text { Injuries }\end{array}$} & None & $7(28.0)$ \\
\cline { 2 - 3 } & Extremities & $21(84.0)$ \\
\cline { 2 - 3 } & Genitourinary & $3(12.0)$ \\
\hline
\end{tabular}

Table-I. Distribution of patients according to demographic profile. $(n=25)$

We noted that most male and female patients had an age $>30$ years as $76.2 \%$ and $50.0 \%$ respectively. There was a greater proportion of hypertension comorbidity in females $3(75.0 \%)$ than male patients $9(42.9 \%)$. According to the Southampton classification, no SSI was recorded in all patients because the majority 21 (84.0\%) had normal healing. Also, no mortality was noted.

The acetabular fractures were evaluated using the Judet and Letournel classification while the pelvic ring fracture using Young-Burgess among all patients and the results indicated that there was a greater proportion of acetabular fractures $(15 / 25=60 \%)$ as compared to pelvic fracture $(10 / 25=40 \%)$ and among these females were more in pelvic ring fracture $(3 / 4=75 \%)$ as compared to males $(8 / 21=38.1 \%)$. Most common fracture type of anterior column and posterior Hemi-transverse, and posterior column and posterior wall in equal proportion of $14.3 \%$ while the same proportion of $14.3 \%$ in the pelvic type of fracture as LC-II and vertical shear was seen in male patients. The results revealed no significant association of Gender with these parameters ( $p>0.05)$, as shown in Table-II and Table-III.

\section{DISCUSSION}

In this study, a total of 25 patients were included diagnosed with pelvi-acetabular fractures.among these majorities were male (84.0\%), while fewer (16.0\%) were female patients with overall mean age of $38.04 \pm 14.58$ years. Male predominance in pelvi-acetabular fracture incidence may be due to the high incidence of the male population on roads and involvement in RTAs, younger population involving high energy trauma more often on streets than older mature community. ${ }^{15}$ An interesting result was the high number of the female population (75.0\%) having pelvic ring injuries as compared to the male population $(38.1 \%)$ and the high number of the male population $(71.9 \%)$ having acetabular fractures as compared to females population (25.0\%). The average stay of patients in the hospital was $5.08 \pm 0.76$ days.

\begin{tabular}{|c|c|c|c|c|c|}
\hline \multicolumn{2}{|c|}{ Attributes } & \multirow{2}{*}{$\begin{array}{c}\text { Total } \\
2 \\
\end{array}$} & \multirow{2}{*}{$\begin{array}{c}\text { Male (\%) } \\
1(4.8)\end{array}$} & \multirow{2}{*}{$\begin{array}{c}\text { Female (\%) } \\
1(25)\end{array}$} & P-Value \\
\hline \multirow[b]{2}{*}{ Age } & $<15$ years & & & & \multirow{2}{*}{0.350} \\
\hline & $15-30$ years & 5 & $4(19)$ & $1(25)$ & \\
\hline \multirow{4}{*}{ Comorbidity } & Smokers & 4 & $4(19)$ & - & \multirow{4}{*}{0.083} \\
\hline & Diabetes Mellitus & 1 & - & $1(25)$ & \\
\hline & $\begin{array}{c}\text { Chronic obstructive } \\
\text { pulmonary disease (COPD) }\end{array}$ & 1 & $1(4.8)$ & - & \\
\hline & Nil & 7 & 7 (33.3) & - & \\
\hline Associated Injuries & None & 21 & $17(81)$ & $4(100)$ & 0.635 \\
\hline
\end{tabular}

Table-II. Stratification of age, comorbidity and associated injuries based on gender distribution. $(n=25)$ 


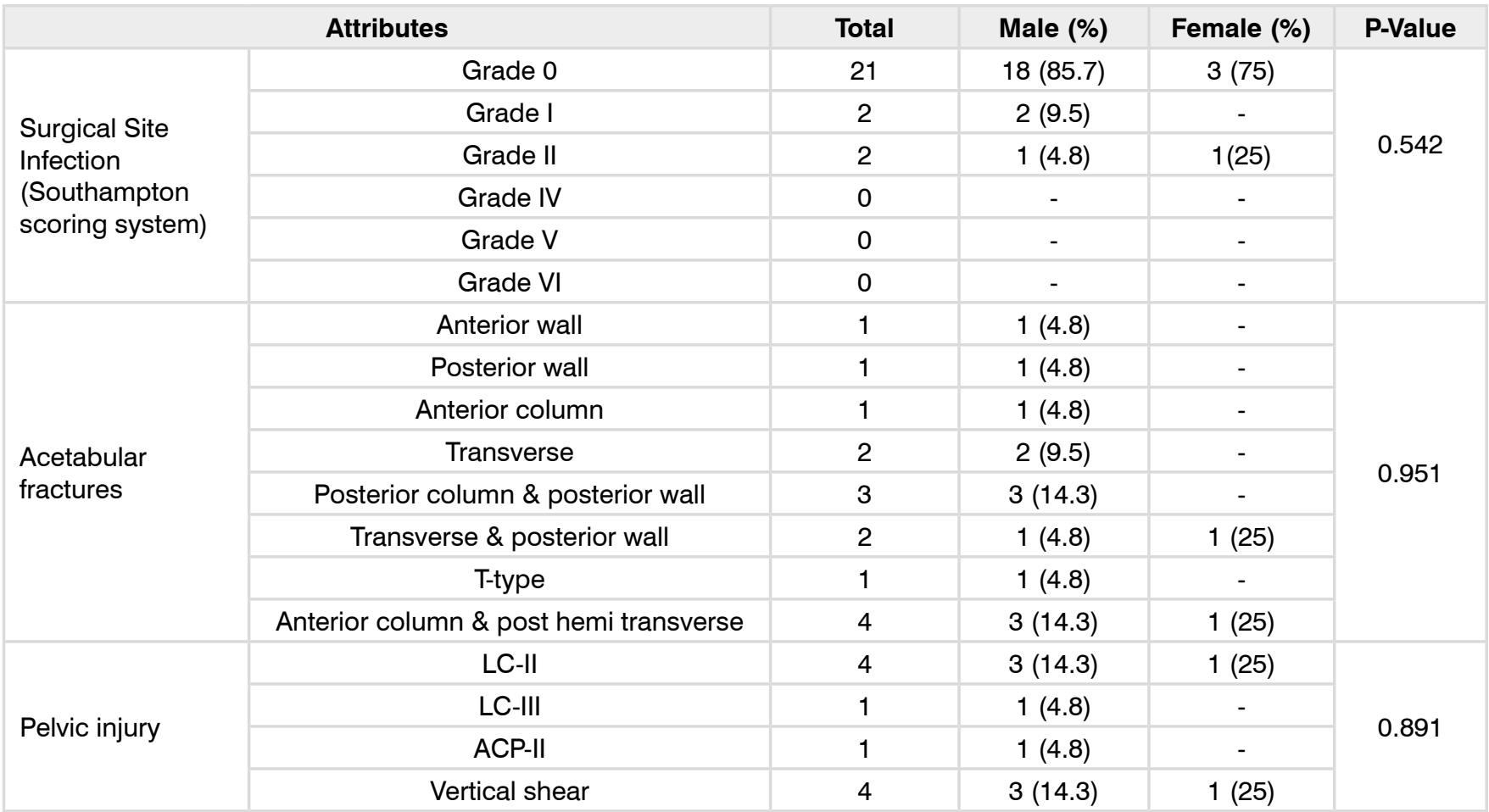

Table-III. Stratification of surgical site infection, fracture site and pelvic injury grading based on gender distribution. $(n=25)$

The hospital's average length of stay is less than most studies because most of the patients were discharged early after initial post-op care to avoid covid 19 contacts in hospital settings. Many studies have proved that the emergent nature of pelvi-acetubar surgery,early internal and external fixation is necessary for early mobilization, improved outcomes, patient survival ${ }^{5}$, and reduced complications. ${ }^{6,7}$ Early fixation avoids anatomic reduction-related problems. ${ }^{8-11}$

Pakistan is a developing country with very limited health resources. Even in the U.K., NHS reported the longest waiting list for surgery since the record begins to introduce precautions to avoid aerosols blamed to be the cause of the corona virus spread during surgical procedures. ${ }^{12}$ Here in Pakistan, the pandemic's panic response could have catastrophic effects on pelvi-acetabular trauma services and already underdeveloped health systems. Therefore, we decided to provide emergency services to pelvi-acetabular trauma patients without any added risk to patients' healthiness during COVID-19 panic situations. According to the Southampton classification, no infection was recorded in all patients because most had normal healing. Also, no mortality was in all patients. Even during the covid 19 pandemic and low mortality, low infection rate shows no increased risk of infection in the pandemic, opposite to the results of a study from Wuhan city. ${ }^{3}$ None of our patients had mortality during this pandemic phase that shows the provision of emergency trauma services for pelvi-acetabular fracture patients during panic and pandemic is nothing to with morbidity and mortality but high fear factor only. A recent study showed that shock response to global calamity and delaying surgeries during the covid 19 pandemics was unnecessary. ${ }^{16}$ In this study, we evaluated and demonstrated our work for managing emergency pelvi-acetabular trauma during the COVID-19 pandemic and the impact of COVID 19 pandemic on the timing of surgery, morbidity, and mortality after pelvi acetabular fixation.

\section{CONCLUSION}

There is no added risk of infection and mortality for emergency trauma services to pelviacetabular fractured patients even during panic 
and pandemic situations. So, Lead the way or leave the way.

Copyright(C 05 Aug, 2021.

\section{REFERENCES}

1. Pakistan prepares to fight back as two coronavirus cases emerge in country". Arab News PK. 26 February 2020. Retrieved 4 March 2020.

2. Khan $H$, Khan MZ, Naveed MM. CoVID-19: An Experience from a Tertiary Care Hospital of Nowshera. Journal of Rawalpindi Medical College. 2020; 24(2):149-55.

3. Guo X, Wang J, Hu D, et al. Survey of COVID-19 disease among orthopaedic surgeons in Wuhan, People's Republic of China. J Bone Joint Surg Am. 2020; 102(10):847-854. doi:10.2106/JBJS.20.00417.

4. Mouton C, Hirschmann MT, Ollivier M, Seil R, Menetrey J. COVID-19-ESSKA guidelines and recommendations for resuming elective surgery. Journal of experimental orthopaedics. 2020; 7:1-7.

5. Corrêa WO, Batista VG, Júnior EF, Fernandes MP, Fortes $\mathrm{R}$, Ruiz GZ, et al. Mortality predictors in patients with pelvic fractures from blunt trauma. Rev Col Bras Cir. 2017; 44(3):222-230.

6. Connor GS, McGwin Jr G, MacLennan PA, Alonso JE, Rue LW. Early versus delayed fixation of pelvic ring fractures. Am Surg. 2003;69(12):1019.

7. Brueton RN. A review of $\mathbf{4 0}$ acetabular fractures: The importance of early surgery. Injury. 1993; 24(3):171-4.

8. Demetriades $\mathrm{D}$, Karaiskakis $\mathrm{M}$, Toutouzas $\mathrm{K}$, Alo $\mathrm{K}$, Vselmahos G, Chan L. Pelvic fractures: Epidemiology and predictors of associated abdominal injuries and outcomes. Journal of the American College of Surgeons. 2002; 195(1):1-0.
9. Vallier HA, Cureton BA, Ekstein C, Oldenburg FP, Wilber $\mathrm{JH}$. Early definitive stabilization of unstable pelvis and acetabulum fractures reduces morbidity. Journal of Trauma and Acute Care Surgery. 2010; 69(3):677-84.

10. Enninghorst N, Toth L, King KL, McDougall D, Mackenzie $S$, Balogh ZJ. Acute definitive internal fixation of pelvic ring fractures in polytrauma patients: $A$ feasible option. Journal of Trauma and Acute Care Surgery. 2010; 68(4):935-41.

11. Ankarath S, Giannoudis PV, Barlow I, Bellamy MC, Matthews SJ, Smith RM. Injury patterns associated with mortality following motorcycle crashes. Injury. 2002; 33(6):473-477.

12. Alton TB, Gee AO. Classifications in brief: Letournel classification for acetabular fractures. Clin Orthop Relat Res. 2014; 472(1):35-38.

13. Manson T, O'Toole RV, Whitney A, Duggan B, Sciadini $M$, Nascone J. Young-Burgess classification of pelvic ring fractures: Does it predict mortality, transfusion requirements, and non-orthopaedic injuries? J Orthop Trauma. 2010; 24(10):603-9.

14. Claroni C, Marcelli ME, Sofra MC, Covotta M, Torregiani G, Giannarelli D, Forastiere E. Preperitoneal continuous infusion of local anesthetics: What is the impact on surgical wound infections in humans? Pain Med. 2016; 17(3):582-589.

15. Melhem E, Riouallon G, Habboubi K, Gabbas M, Jouffroy P. Epidemiology of pelvic and acetabular fractures in France. Orthop Traumatol Surg Res. 2020; 106(5):831-839.

16. Cook TM. Personal protective equipment during the coronavirus disease (COVID) 2019 pandemic - A narrative review. Anaesthesia. 2020; 75(7):920-927. 


\section{AUTHORSHIP AND CONTRIBUTION DECLARATION}

\begin{tabular}{|c|l|l|l|}
\hline No. & Author(s) Full Name & \multicolumn{1}{|c|}{ Contribution to the paper } & Author(s) Signature \\
\hline 1 & Umair Nadeem & Literature Review, Methodology. & \\
\hline 2 & Atif Ali & Data Collection, Discussion. & \\
3 & Nayab Shakoor & Literature review, Data analysis. \\
\hline 4 & Jawad Hafeez & Data collection. \\
\hline 5 & Majid Zaheer & Introduction, References. \\
\hline 6 & Zubair Khalid & Drafting, Proof reading. \\
7 & Ameer Aziz & Final approval, Supervision. & \\
\hline
\end{tabular}

